\title{
Tratamiento quirúrgico del hipospadias. Experiencia de 10 años
}

\section{Surgical treatment of hypospadias. Ten-year experience}

\author{
Adrián Alejandro González-Maldonado, ${ }^{1}$ Gildardo Manzo-Pérez, ${ }^{1}$ Marco Antonio Vanzzini-Guerrero, ${ }^{1}$ Braulio \\ Omar Manzo-Pérez, ${ }^{2}$ Edgard Efrén Lozada-Hernández, ${ }^{3}$ Héctor Manuel Sánchez-López ${ }^{4}$
}

\begin{abstract}
Resumen
ANTECEDENTES: El hipospadias es la segunda anomalía congénita urológica más frecuente en varones recién nacidos. En México se ha reportado una prevalencia de 2.6 por cada 10,000 nacimientos. Aunque existen diversas técnicas quirúrgicas, ninguna se ha estandarizado como tratamiento de elección.

OBJETIVO: Analizar la experiencia de 10 años en el tratamiento de hipospadias, además de describir sus características, técnicas quirúrgicas y complicaciones.

MATERIALES Y MÉTODOS: Estudio descriptivo, retrospectivo, observacional y transversal al que se incluyeron pacientes operados del hipospadias de 2008 a 2017. Evaluación y análisis de las características del hipospadias, técnicas quirúrgicas implementadas, complicaciones a corto y largo plazo, y tasa de éxito. Las variables categóricas se reportaron como frecuencia y porcentaje. Reporte de variables categóricas: frecuencia y porcentaje. Comparación de variables cualitativas con $\chi^{2}$ o prueba exacta de Fisher. Estimación de medidas de asociación mediante el cálculo de razón de momios e intervalo de confianza de 95\%. Se consideró estadísticamente significativo el valor de $\mathrm{p}<0.05$. RESULTADOS: Se registraron 190 pacientes en quienes se efectuó la corrección del hipospadias. De acuerdo con la localización del meato uretral, el hipospadias se clasificó en distal (42.6\%), medio (29\%) y proximal (28.4\%). La edad media fue de 38.9 \pm 30.6 meses. La técnica quirúrgica más utilizada fue la tubulización con incisión del plato $(46.3 \%)$. Se reportaron complicaciones posoperatorias en $52.1 \%$ de los casos, principalmente fístula uretrocutánea (24.2\%), asociada con la técnica de tubulización con incisión del plato (38.9\%), seguida de Thiersch-Duplay (20.3\%), Onlay (16.9\%), Duckett (13.5\%), glanduloplastia de aproximación (5.08\%), Mathieu (3.3\%) y avance meatal con glanduloplastia (1.6\%). La dehiscencia del glande ocurrió en $15.7 \%$ y estenosis del meato en $7.8 \%$.
\end{abstract}

CONCLUSIONES: La corrección del hipospadias y sus complicaciones siguen siendo un reto para los cirujanos. Este estudio sirve de precedente para identificar las fallas y debilidades al momento de efectuar la corrección quirúrgica. La identificación de factores asociados con la disminución de las complicaciones permanece en investigación.

PALABRAS CLAVE: Hipospadias; complicaciones; técnicas quirúrgicas; tubulización con incisión del plato.

\section{Abstract}

BACKGROUND: Hypospadias is the second most frequent congenital anomaly in newborn males and its frequency in Mexico is reported at 2.6 per 10,000 births. Despite the fact that hundreds of surgical techniques have been described, none of them have been standardized as ideal treatment.

OBJECTIVE: The aim of the present study was to analyze our 10 years of experience in the surgical treatment of hypospadias and describe its characteristics, surgical techniques, and complications.

MATERIALS AND METHODS: We conducted a retrospective, descriptive, observational, and cross-sectional study on the patients that underwent hypospadias repair over the past 10 years (2008-2017). The characteristics of the hypospadias, surgical techniques employed, short-term and long-term complications, and success rates were evaluated. The categorical variables were reported as frequency and percentage and the qualitative
${ }^{1}$ Servicio de Nefro-Urología y Trasplante, Departamento de Urología Pediátrica.

${ }^{2}$ Servicio de Nefro-Urología y Trasplante, Departamento de Urología. ${ }^{3}$ Jefe del servicio de Cirugía General. ${ }^{4}$ Jefe del servicio de Nefro-Urología y Trasplante.

Hospital Regional de Alta Especialidad del Bajío, León, Guanajuato.

Recibido: abril 2018

Aceptado: julio 2018

Correspondencia

Adrián Alejandro González Maldonado dr.adrian@outlook.com

Este artículo debe citarse como González-Maldonado AA, Manzo-Pérez G, Vanzzini-Guerrero MA, Manzo-Pérez $B O$, Lozada-Hernández EE, SánchezLópez HM. Tratamiento quirúrgico del hipospadias. Experiencia de 10 años. Rev Mex Urol. 2018 julio-agosto;78(4):263-272.

DOI: https://doi.org/10.24245/revmexurol.v78i4.2129 
variables were compared using the chi-square test or the Fisher's exact test, as required. Measures of association were calculated through the odds ratio with a 95\% confidence interval. Statistical significance was set at a $p<0.05$.

RESULTS: We analyzed the data of 190 patients that underwent hypospadias repair. The pathology was classified in accordance with urethral meatus location as distal (42.6\%), medial $(29 \%)$, and proximal $(29 \%)$. Mean patient age was $38.9 \pm 30.6$ months. The most widely used surgical technique was tubularization with incision of the urethral plate $(46.3 \%)$. Postoperative complications presented in $52.1 \%$, mainly urethrocutaneous fistula $(24.2 \%)$ associated with the tubularized incised plate technique $(38.9 \%)$, followed by the Thiersch-Duplay $(20.3 \%)$, onlay (16.9\%), Duckett $(13.5 \%)$, glans approximation procedure $(5.08 \%)$, Mathieu $(3.39 \%)$, and meatal advancement with glanuloplasty (1.69\%) techniques. Dehiscence of the glans penis presented in $15.79 \%$ of the patients and meatal stricture in $7.89 \%$.

CONCLUSIONS: Hypospadias surgery and its complications continue to be a surgical challenge. The present study serves as a precedent for identifying failures and weaknesses at the time of carrying out surgical corrections and the identification of factors associated with complication reduction continues to be studied.

KEYWORDS: Hypospadias; Complications; Surgical techniques; Tubularized incised plate.

\section{ANTECEDENTES}

El hipospadias es una alteración congénita que resulta del desarrollo anormal del pene, debido a un defecto de la estimulación andrógena. ${ }^{1}$ Representa la segunda anomalía congénita urológica más frecuente en varones recién nacidos (después de los testículos no descendidos); su incidencia es de 1 por cada 125-300 nacimientos..$^{2-4}$ Es la consecuencia de varios defectos en la uretra, cuerpo esponjoso y cuerpos cavernosos, y se caracteriza por la abertura proximal de la uretra, curvatura peneana, coexistencia de capuchón y ausencia ventral del prepucio..$^{2,4}$ La prevalencia (por 10,000 nacimientos vivos) estimada en Europa es de 19.9 (límites: 1-464), Estados Unidos 34.2 (6-129.8), Sudamérica 5.2 (2.8-110), Asia 0.6-69, África 5.9 (1.9-110) y Australia 17.1 (34.8). ${ }^{5}$ En México se ha reportado una prevalencia de 2.6 por 10,000 nacimientos. ${ }^{6}$

La clasificación de referencia de hipospadias se basa en la ubicación del meato uretral, ya sea distal (anterior), medio o proximal (posterior). Ocasionalmente puede referirse como leve a los hipospadias distales y graves a los proximales; sin embargo, este sistema de clasificación es engañoso. Algunas variantes de hipospadias anteriores se asocian con curvatura peneana, lo que puede requerir una reconstrucción quirúrgica más difícil, mientras que algunos casos aparentemente graves de hipospadias proximales no representan un desafío quirúrgico cuando la anatomía es favorable. ${ }^{7}$ En $70 \%$ de los casos el meato uretral se localiza distal en el pene y en $30 \%$ restante tiene una localización proximal. ${ }^{2}$

Galeno fue el primero en describir el hipospadias (130-199 dC), haciendo énfasis en la curvatura del pene. Casi 1500 años después, Ambrosio Paré (1510-1590), considerado el padre de la cirugía, señaló esta alteración en uno de sus libros. ${ }^{8} \mathrm{~A}$ partir de entonces se ha descrito ampliamente esta anomalía y se han reportado más de 300 técnicas quirúrgicas. No obstante, la hipospadiología moderna evolucionó considerablemente desde la década de 1980 y aún persiste el reto por mejorar los resultados a largo plazo. ${ }^{8-10}$

La elección de la técnica depende, principalmente, de la experiencia del cirujano en diferentes tipos de hipospadias; sin embargo, 
debe ser capaz de definir bien los componentes anatómicos, y si se requiere, elegir y modificar la técnica de acuerdo con la anatomía. Actualmente la técnica de tubulización del plato uretral, descrita por Thiersch y Duplay en 1869 y 1874 , respectivamente, y más tarde modificada por Snodgrass (1994) con incisión del plato uretral (TIP), es una de las más utilizadas por sus buenos resultados..$^{8,11,12}$

La finalidad del procedimiento quirúrgico del hipospadias es enderezar el pene, lograr llevar el nuevo meato lo más distal posible y conseguir una apariencia y función normal. .,4 La corrección puede efectuarse de manera temprana. Hoy día la Academia Americana de Pediatría y la Asociación Europea de Urología recomiendan realizar el procedimiento entre los 6 y 18 meses de edad. Estas recomendaciones se basan en consideraciones psicológicas, quirúrgicas y anestésicas. ${ }^{2,9}$

De acuerdo con algunos estudios, se obtienen mejores resultados quirúrgicos en pacientes con hipospadias distal que proximal, con $5-10 \%$ de complicaciones. En sujetos con hipospadias proximal se han reportado complicaciones de $15-50 \%$ de los casos. ${ }^{7,13}$ Para clasificar las complicaciones posoperatorias se utiliza la escala de Clavien-Dindo. ${ }^{14}$

Las complicaciones posoperatorias pueden identificarse de manera temprana en los primeros meses de la cirugía. No obstante, se sugiere el seguimiento a largo plazo, porque puede aparecer de forma tardía una fístula uretrocutánea o haber recurrencia de la curvatura. Las complicaciones más frecuentes incluyen: formación de fístulas uretrocutáneas, estenosis del meato, estrechez de la uretra, dehiscencia del glande, formación de un divertículo uretral, alteraciones cosméticas y recurrencia de la curvatura. Incluso se ha reportado el desarrollo de vello en la uretra, disfunción eréctil y balanitis xerótica obliterante. ${ }^{7,15}$
El objetivo del estudio fue: describir las características del hipospadias, técnicas quirúrgicas utilizadas para su corrección, complicaciones a corto y largo plazo, tasa de reintervención y complicaciones asociadas.

\section{MATERIALES Y MÉTODOS}

Estudio descriptivo, retrospectivo, observacional y transversal, llevado a cabo en pacientes con diagnóstico de hipospadias, intervenidos en el Hospital Regional de Alta Especialidad del Bajío, en León, Guanajuato, de enero de 2008 a enero de 2017. Criterios de inclusión: pacientes con hipospadias distal, medio y proximal. Criterios de exclusión: sujetos en quienes no se especificó la técnica quirúrgica utilizada, que se intervinieron inicialmente en otro hospital y quienes no acudieron al seguimiento. Los pacientes fueron intervenidos por tres cirujanos urólogos-pediatras. Los resultados de las cirugías se evaluaron por el número de complicaciones identificadas durante el seguimiento.

Para el análisis estadístico se utilizó el programa SPSS v. 21. Se realizó estadística descriptiva para conocer la distribución de las variables cuantitativas. Los resultados con criterios de normalidad se reportaron como media y desviación estándar, y sin normalidad como mediana y rango intercuartilar $25-75 \%$. Las variables categóricas se reportaron como frecuencia y porcentaje. Las variables cualitativas se compararon con la prueba de $\chi^{2}$ o exacta de Fisher, según fuera el caso. Se estimaron medidas de asociación a través del cálculo de la razón de momios (RM), con intervalo de confianza de 95\% (IC95\%). Se consideró estadísticamente significativo el valor de $\mathrm{p}<0.05$.

\section{RESULTADOS}

La muestra analizada fue de 190 pacientes. La edad media al momento de la cirugía fue de 38.9 
土 30.66 meses (límite mínimo de 6 y máximo 176 meses) y mediana de 28 meses. Con base en los datos obtenidos se clasificaron los hipospadias, según su localización, en distal (42.6\%), medio (28.9\%) y proximal (28.4\%) (Figura 1). Entre las anormalidades asociadas con hipospadias se encontraron: testículo no descendido (19.1\%), coexistencia de capuchón (30\%) y cuerda (42.6\%), de grado leve $\left(30^{\circ}\right)$, moderado $\left(45^{\circ}\right)$ y severo $\left(90^{\circ}\right)$ en $21,11.5$ y $10 \%$, respectivamente (en $57.3 \%$ de los pacientes no se reportó cuerda).

La técnica quirúrgica utilizada con mayor frecuencia fue la tubulización con incisión del plato uretral (TIP) en $46.3 \%$ (Figura 2). A todos los pacientes con hipospadias medio y proximal se les realizó una erección artificial con solución salina para registrar la coexistencia de cuerda peneana y valorar su gravedad. De los pacientes con hipospadias proximal (28.4\%), en $22.2 \%$ se Ilevó a cabo la cirugía en dos tiempos, debido a la gravedad de la cuerda, utilizando mucosa de prepucio en 8 casos y oral en 4 para reconstrucción del plato uretral en el primer tiempo; por su parte, en 1 caso con hipospadias medio se utilizó mucosa oral. El tiempo entre la primera y la segunda cirugía fue de $12.03 \pm 8.62$ meses (límite mínimo de 6 y máximo de 37 meses).

\section{Seguimiento}

Se colocó un catéter uretral de silicona, calibre 6,8 y $10 \mathrm{Fr}$ en $31.5,44.2 \%$ y $4.2 \%$ de los casos, respectivamente. Dos semanas después del egreso hospitalario se concretó una cita médica de control para el retiro de la sonda, que en promedio permaneció 14 días. La siguiente cita fue un mes después, para valorar el estado posoperatorio. El seguimiento tuvo una media de 21.9 meses (límite mínimo de 1 y máximo de 98 meses) y mediana de 9 meses. En 56.3\% de los pacientes se reportó un seguimiento mayor de 12 meses, en $33.6 \%$ mayor de 24 meses, en $20 \%$ mayor de 36 meses, en $10 \%$ mayor de

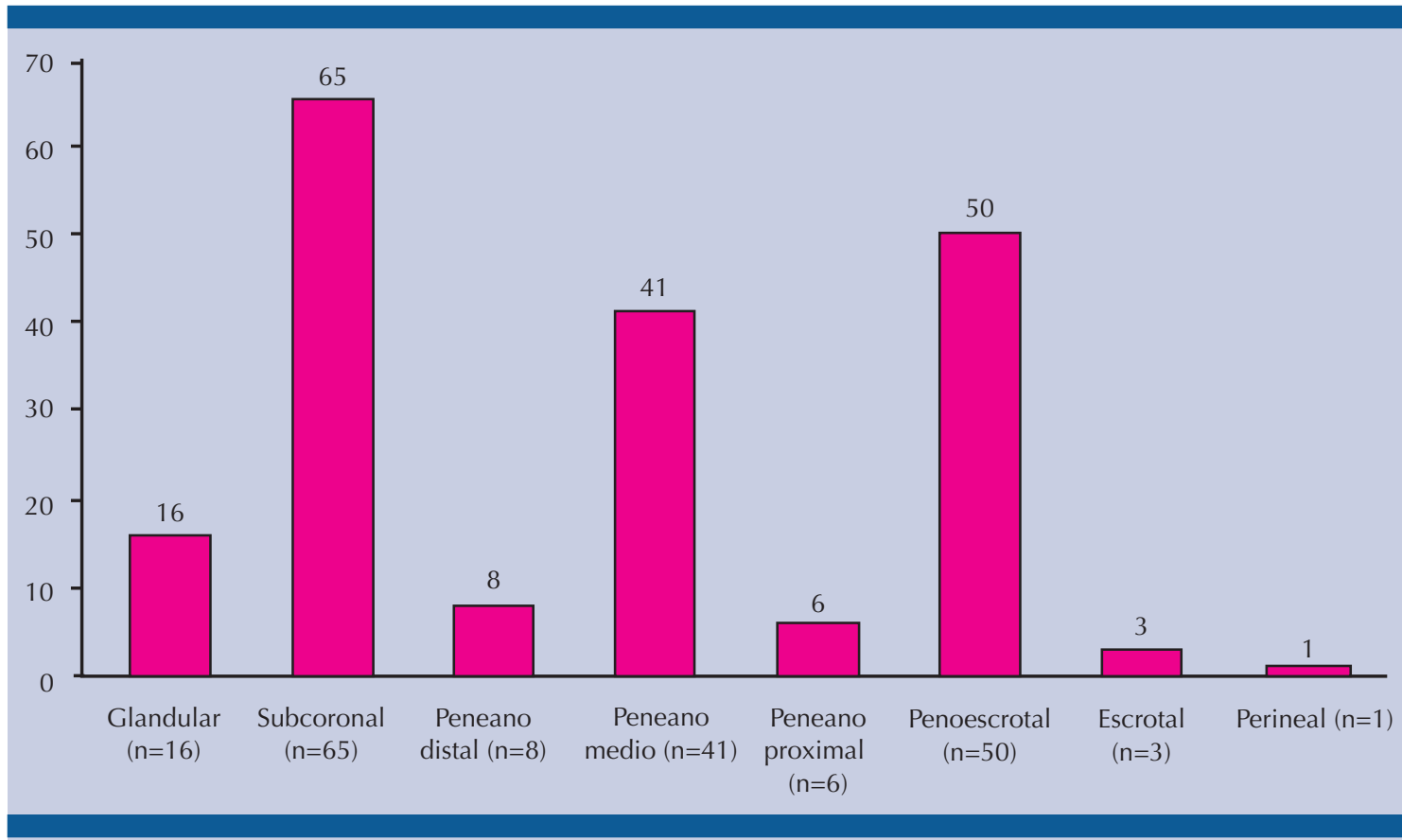

Figura 1. Localización del meato uretral. 


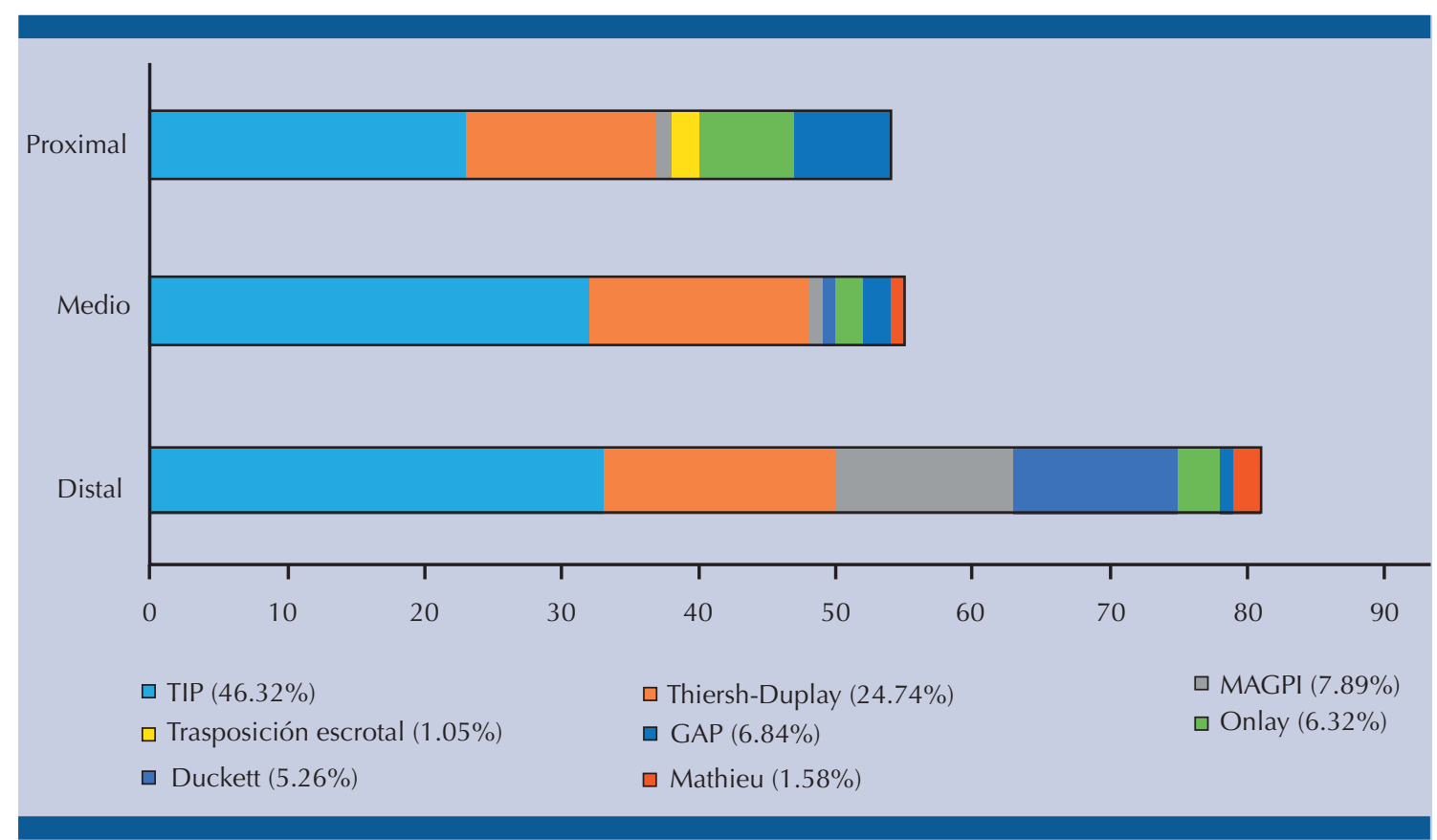

Figura 2. Técnicas de reparación del hipospadias.

TIP: tubulización con incisión del plato, GAP: glandulosplastia de aproximación, MAGPI: avance meatal con glandulosplastia.

48 meses y en $4.73 \%$ mayor de 60 meses del posoperatorio.

\section{Complicaciones}

Se encontraron complicaciones posoperatorias (Cuadro 1, Figura 3) en 52.1\% (grado I de la clasificación Clavien-Dindo: $17.1 \%$; grado II, $1 \%$ y grado IIIb, $81.8 \%$ ), principalmente fístula uretrocutánea (24.2\%). El 17.6\% de los casos reportó dos o más complicaciones. Las complicaciones aparecieron después de 48 días (mediana), con un percentil de $25-75 \%$ de 20-134 días, posterior a la cirugía definitiva. En relación con los pacientes a quienes se efectuó tubulización con incisión del plato uretral, la fístula uretrocutánea también fue la complicación más frecuente (26.1\%). Cuadro 2, Figura 4

El Cuadro 3 muestra las técnicas y complicaciones identificadas en el estudio, entre las que destacan: tubulización con incisión del plato (Figura 2), seguida de la técnica de Thiersch-Duplay, el colgajo transverso de la isla del prepucio Onlay, Duckett, GAP (por sus siglas en inglés: glans approximation procedure, procedimiento de aproximación del glande) y MAGPI (por sus siglas en inglés: meatal advancement and glanuloplasty, avance meatal y glanduloplastia).

La técnica quirúrgica con mayor número de complicaciones posoperatorias fue el colgajo transverso de la isla del prepucio Onlay (83.3\%), le siguió la de Duckett (80\%), técnica TIP (59\%), Thiersch-Duplay (46.8\%), GAP (23\% ) y MAGPI (13.3\%).

La fístula uretrocutánea fue la complicación más frecuente $(52.5 \%)$, sobre todo en pacientes en quienes se utilizó la técnica TIP (38.9\%), seguida de la técnica Thiersch-Duplay (20.3\%), Onlay $(16.9 \%)$, Duckett $(13.5 \%)$, GAP (5\%), Mathieu 
Cuadro 1. Técnicas quirúrgicas y complicaciones

\begin{tabular}{|l|c|c|c|c|c|c|}
\hline & \multicolumn{5}{|c|}{ Complicaciones } & Total \\
\cline { 2 - 6 } & $\begin{array}{c}\text { Fístula } \\
\text { uretrocutánea }\end{array}$ & $\begin{array}{c}\text { Estenosis del } \\
\text { meato uretral }\end{array}$ & $\begin{array}{c}\text { Divertículo } \\
\text { uretral }\end{array}$ & $\begin{array}{c}\text { Dehiscencia } \\
\text { del glande }\end{array}$ & $\begin{array}{c}\text { Necrosis } \\
\text { de piel }\end{array}$ & \\
\hline TIP & $23(38.9 \%)$ & $8(72.7 \%)$ & $1(25 \%)$ & $20(83.3 \%)$ & - & $52(52.5 \%)$ \\
\hline Thiersch-Duplay & $12(20.3 \%)$ & $3(27.2 \%)$ & $3(75 \%)$ & $3(12.5 \%)$ & $1(100 \%)$ & $22(22.2 \%)$ \\
\hline Onlay & $10(16.9 \%)$ & - & - & - & - & $10(10.1 \%)$ \\
\hline Duckett & $8(13.5 \%)$ & - & - & - & - & $8(8.1 \%)$ \\
\hline GAP & $3(5.1 \%)$ & - & - & - & - & $3(3 \%)$ \\
MAGPI & $1(1.6 \%)$ & - & - & $1(4.1 \%)$ & - & $2(2 \%)$ \\
\hline Mathieu & $2(3.3 \%)$ & - & - & - & - & $2(2 \%)$ \\
Total & $\mathbf{5 9}$ & $\mathbf{1 1}$ & $\mathbf{4}$ & $\mathbf{2 4}$ & $\mathbf{1}$ & $\mathbf{1 0 0}$
\end{tabular}

TIP: tubulización con incisión del plato, GAP: glanduloplastia de aproximación, MAGPI: avance meatal con glanduloplastia.

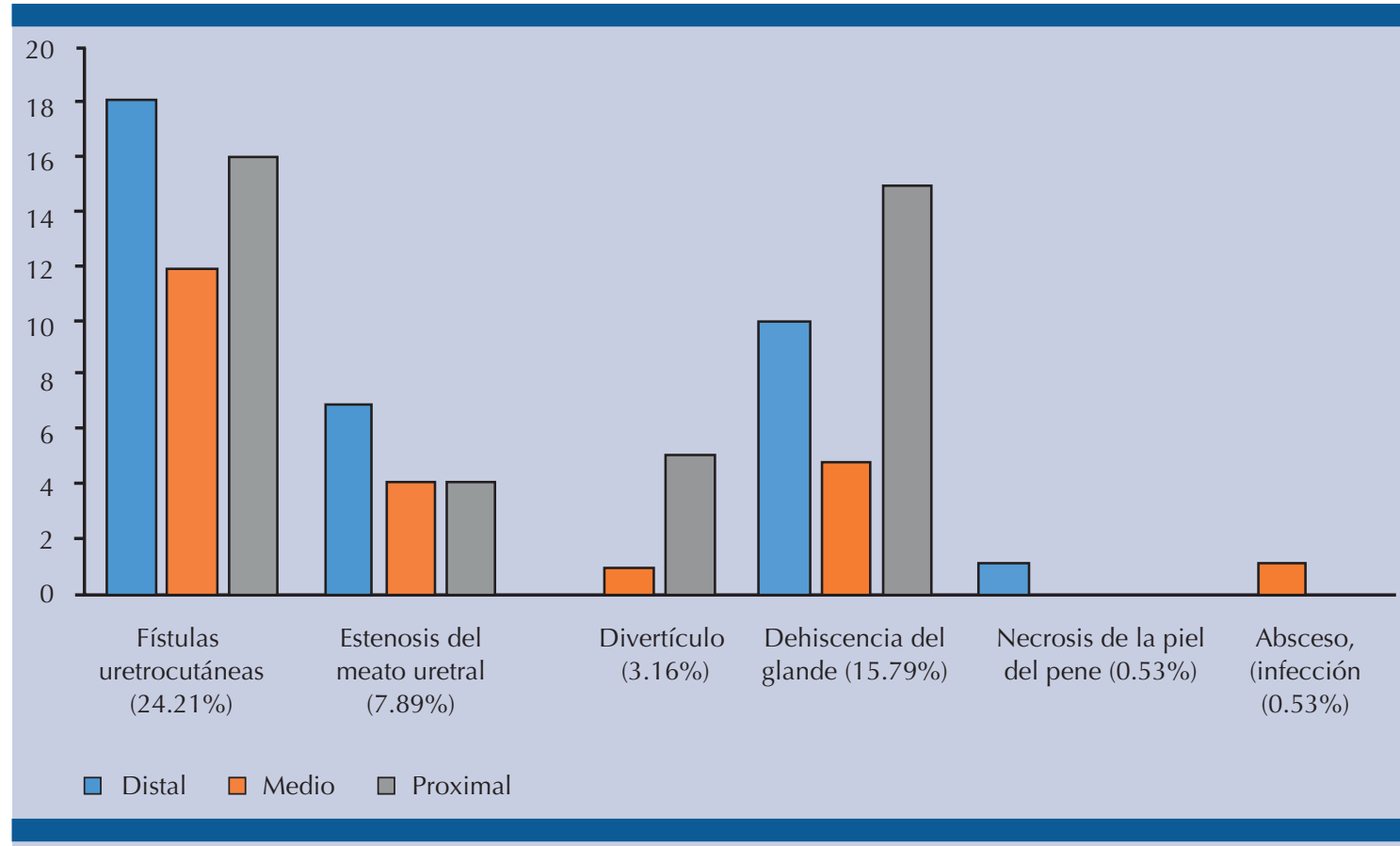

Figura 3. Complicaciones de la reparación quirúrgica del hipospadias $(p=0.004)$.

(3.3\%) y MAGPI (1.6\%) (Cuadro 1). La estenosis del meato uretral apareció en sujetos a quienes se practicó la técnica TIP y Thiersch-Duplay (72.7 vs $27.2 \%$, respectivamente). El divertículo uretral también se encontró asociado con ambas técnicas, pero se registraron más casos en pacientes con la técnica Thiersch-Duplay (25 vs 75\%, respetivamente). La dehiscencia del glande se reportó en $83.3 \%$ de los casos con técnica TIP, $12.5 \%$ en Thiersch-Duplay y $4.1 \%$ en MAGPI. La necrosis de piel fue reportada en 1 caso de Thiersch-Duplay. 
Cuadro 2. Incisión y tubulización del plato uretral

\begin{tabular}{|l|c|c|}
\hline Variables & $\mathbf{n}$ & $\%$ \\
\hline Total de casos & 88 & 46.3 \\
\hline Hipospadias distal & 33 & 37.5 \\
\hline Hipospadias medio & 32 & 36.3 \\
\hline Hipospadias proximal & 23 & 26.1 \\
\hline Complicaciones & & \\
\hline Fístula uretrocutánea & 23 & 26.1 \\
\hline Dehiscencia del glande & 20 & 22.7 \\
\hline Estrechez uretral & 8 & 9.1 \\
\hline Divertículo & 1 & 1.1
\end{tabular}

Cuadro 3. Complicaciones posquirúrgicas y su relación con la técnica y la posición del meato uretral

\begin{tabular}{|c|c|c|c|}
\hline \multirow[t]{2}{*}{ Técnica quirúrgica } & \multicolumn{2}{|c|}{ Complicaciones } & \multirow{2}{*}{$\frac{p}{0.0005}$} \\
\hline & Sí & No & \\
\hline TIP & $\begin{array}{c}52 \\
(52.5 \%)\end{array}$ & $36(39.5 \%)$ & \\
\hline Thiersch-Duplay & $\begin{array}{c}22 \\
(22.2 \%)\end{array}$ & $25(27.4 \%)$ & \\
\hline $\begin{array}{l}\text { Colgajo transverso } \\
\text { de isla de prepucio } \\
\text { Onlay }\end{array}$ & $\begin{array}{c}10 \\
(10.1 \%)\end{array}$ & $2(2.2 \%)$ & \\
\hline Duckett & $8(8.1 \%)$ & $2(2.2 \%)$ & \\
\hline GAP & $3(3 \%)$ & $10(10.9 \%)$ & \\
\hline MAGPI & $2(2 \%)$ & $13(14.2 \%)$ & \\
\hline Mathieu & $2(2 \%)$ & $1(1.1 \%)$ & \\
\hline $\begin{array}{l}\text { Transposición } \\
\text { penoescrotal }\end{array}$ & - & $2(2.2 \%)$ & \\
\hline $\begin{array}{l}\text { Posición del meato } \\
\text { uretral }\end{array}$ & Sí & No & 0.001 \\
\hline Distal & $\begin{array}{c}36 \\
(36.3 \%)\end{array}$ & $45(49.4 \%)$ & \\
\hline Medio & $\begin{array}{c}23 \\
(23.2 \%)\end{array}$ & $32(35.1 \%)$ & \\
\hline Proximal & $\begin{array}{c}40 \\
(40.4 \%)\end{array}$ & $14(15.3 \%)$ & \\
\hline
\end{tabular}

TIP: tubulización con incisión del plato, GAP: glandulosplastia de aproximación, MAGPI: avance meatal con glandulosplastia.
El hipospadias proximal o posterior fue el tipo con mayores complicaciones $(40.4 \%)$, seguido del hipospadias distal o anterior $(36.3 \%$ ) y el medio (23.2\%). Cuadro 3

Se reintervinieron 83 (43.6\%) niños: $37.3 \%$ en una ocasión, $3.6 \%$ en 2 y $2.63 \%$ en 3 o más ocasiones.

\section{DISCUSIÓN}

Comparada con otros tipos de cirugías reconstructivas, el procedimiento quirúrgico del hipospadias representa una alta tasa de complicaciones. La incidencia estimada varía de 6 a 49\%, según la gravedad del hipospadias. ${ }^{16,17}$

En nuestro estudio, $52.1 \%$ de los pacientes reportó una complicación (de acuerdo con la clasificación de Clavien-Dindo), principalmente en los hipospadias proximales $(40.4 \%, p=0.001) .{ }^{14}$ Este tipo de hipospadias sigue siendo un reto en todo el mundo, pues genera más complicaciones. Long y sus coautores reportaron $13 \%$ ( $n=665$ niños) de pacientes con hipospadias proximal y de éstos, $45 \%$ tuvo algún tipo de complicación. Otros estudios señalan, incluso, 30-68\% de complicaciones en este tipo de hipospadias. ${ }^{13,17}$

De acuerdo con nuestros resultados, la complicación más frecuente fue la fístula uretrocutánea $(24.1 \%)$, principalmente en pacientes con hipospadias distales, seguida de la dehiscencia del glande (15.7\%) en hipospadias proximales y estenosis del meato (7.8\%) en los casos de hipospadias distales. El divertículo se reportó en pacientes con hipospadias medios y proximales.

La bibliografía reporta mayor incidencia de hipospadias distales (36.4-81\%), seguido de hipospadias medios (13-48.6\%) e hipospadias proximales (5-6\%). ${ }^{18-21}$ En este estudio se reportan hipospadias distal, medio y proximal en $42.6,28.9$ y $28.4 \%$, respectivamente. En cuanto 


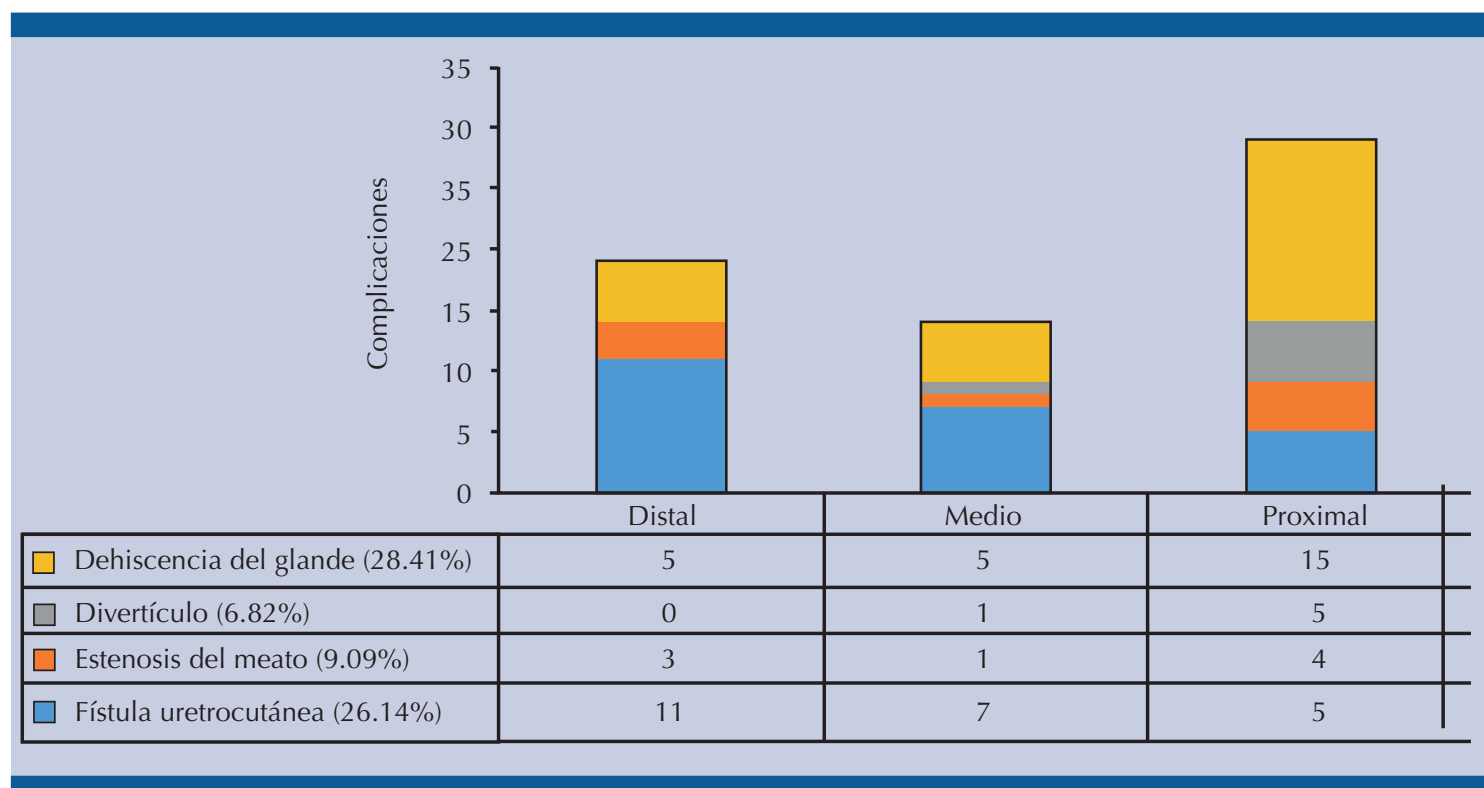

Figura 4. Complicaciones posoperatorias de la tubulización con incisión del plato asociada con la localización del meato uretral.

a las anormalidades, la asociación con cuerda peneana descrita en la bibliografía es de $74.3 \%$ $y$ en nuestro estudio de $42.6 \% .{ }^{17,21}$

Con base en las técnicas quirúrgicas se han reportado diversos tipos, debido a que no todas ofrecen buenos resultados en todos los tipos de hipospadias. ${ }^{10}$ La tubulización con incisión del plato se ha convertido en la técnica de elección desde su introducción, debido a su versatilidad en la corrección de hipospadias, sin importar su localización. ${ }^{12,22}$ En la actualidad, en varios centros hospitalarios prefieren esta técnica para los hipospadias distales por sus buenos resultados. La incidencia de fístulas uretrocutáneas (como la complicación más frecuente) asociadas con la tubulización con incisión del plato es de 3.5$29 \%$ de los casos. ${ }^{10,12,20-27}$ En nuestro estudio, las fístulas aparecieron en $24.2 \%$, incidencia por debajo del límite superior reportado. En nuestra institución implementamos esta técnica en pacientes con hipospadias distales y en menor frecuencia para los medios y proximales. De acuerdo con los resultados, se encontró fístula uretrocutánea en $26.1 \%$ de los pacientes a quienes se efectuó la técnica de tubulización con incisión del plato. Hoy día se prefiere realizar el procedimiento quirúrgico en dos tiempos en pacientes con hipospadias proximales; sin embargo, no existe un consenso de la técnica ideal en pacientes con este tipo de hipospadias, pero si la anatomía lo permite, puede realizarse en un solo tiempo con la técnica de mayor destreza o preferida por el cirujano. ${ }^{9,24}$ En este estudio, el procedimiento quirúrgico en 2 tiempos fue efectuado en 13 pacientes, con preferencia por el uso de mucosa de prepucio $(n=8)$ y tasa de complicación de $61 \%$.

La edad óptima para realizar la corrección del hipospadias es entre los 6 y 18 meses de edad, pues antes de este tiempo existe mayor riesgo para el procedimiento anestésico. ${ }^{4,9,28,29}$ Perlmutter y sus colaboradores reportaron que la cirugía entre los 
4 y 6 meses de edad se asocia con menor tasa de complicaciones..$^{10,30}$ En el Hospital Regional de Alta Especialidad del Bajío la edad óptima para llevar a cabo la primera intervención quirúrgica es a los 6 meses de edad; no obstante, de acuerdo con lo reportado por Arreola-García y su grupo, efectuar la reparación antes de los 18 meses de edad resulta complicado, debido a la demanda de atención de otro tipo de padecimientos urológicos que existen en nuestra población..$^{3,4} \mathrm{El}$ $97.8 \%$ de las cirugías se practican en pacientes mayores de 6 meses ( $38.9 \pm 30.6$ meses). En los casos a quienes se efectuó entre los 6 y 18 meses se registraron $58 \%$ de complicaciones versus $50 \%$ en mayores de 18 meses $(p=0.409$ ).

La composición del material y la técnica de colocación de la sutura pueden contribuir de manera significativa a la corrección del hipospadias. La aplicación de suturas de polidioxanona 7/0 para puntos subcuticulares de la uretroplastia ha demostrado disminución del riesgo de fístulas, comparado con vicryl 6/0.31

La mayor parte de las complicaciones se identifica un año después de la cirugía (usualmente el seguimiento es de dos años). ${ }^{17,31}$ En nuestro hospital, el seguimiento en consulta es de 22 meses (mínimo 1 y máximo 98 meses), con una mediana de 9 meses. En algunos casos los pacientes no acuden a las citas y su diagnóstico se prolonga. ${ }^{9,22}$

\section{CONCLUSIONES}

La intervención quirúrgica por hipospadias y sus complicaciones siguen siendo un reto para los cirujanos. Hasta la fecha no existen métodos estandarizados para la corrección, debido a la variabilidad de defectos y las diferentes técnicas que el cirujano debe conocer para ser capaz de cambiar la decisión y modificar el procedimiento. Este estudio sirve de precedente para identificar las fallas y debilidades al momento de efectuar la corrección quirúrgica. La técnica de elección en este hospital es la tubulización con incisión del plato, que aunque es fácil de realizar, se asocia con diversas complicaciones. La técnica con menor incidencia de complicaciones (46.8 vs 59.1\%) y mejor pronóstico fue la de ThierschDuplay, que es una tubulización sin incisión del plato. Así mismo, el hipospadias proximal se asocia con mayor riesgo de complicaciones; por tanto, es importante continuar con la búsqueda de factores que permitan disminuirlas, así como llevar a cabo la intervención quirúrgica a una edad más temprana, pues la media fue casi del doble de la edad óptima para efectuar la corrección; sin embargo, esta variable es difícil de modificar, debido al sistema de salud en el que estamos inmersos: no obstante, podría disminuir la incidencia de complicaciones en nuestra población. El seguimiento a largo plazo puede ser una variable importante de análisis para identificar complicaciones tardías. Aunque existen más de 300 técnicas para la reparación del hipospadias, el resultado exitoso depende, principalmente, de la capacidad del cirujano y disponibilidad del tejido apropiado para la reparación.

\section{Conflictos de interés}

No existe conflicto de interés.

\section{Fuente de financiamiento}

Esta investigación no recibió ninguna beca específica de agencias de los sectores público, comercial, o sin fines de lucro.

\section{REFERENCIAS}

1. Akbiyik $F$, et al. Clinical experience in hypospadias: results of tubularized incised plate in 496 patients. DOI: https:// doi.org/10.1016/j.urology.2008.06.075

2. van der Horst $\mathrm{HJ}$, de Wall LL. Hypospadias, all there is to know. DOI: 10.1007/s00431-017-2864-5

3. Arreola-García J, et al. Tratamiento quirúrgico del hipospadias y sus complicaciones en relación con la edad del niño. Cir Cir 2014;82(2):157-62.

4. Duckett JW. Hypospadias. Pediatr Rev 1989;11(2):37-42. 
5. Springer A, et al. Worldwide prevalence of hypospadias. DOI: https://doi.org/10.1016/j.jpurol.2015.12.002

6. Källén B, et al. A joint international study on the epidemiology of hypospadias. Acta Paediatr Scand Suppl 1986;324:1-52.

7. Keays MA, Dave S. Current hypospadias management: Diagnosis, surgical management, and long-term patient-centred outcomes. DOI: http://dx.doi.org/10.5489/cuaj.4386

8. Smith ED. The history of hypospadias. Pediatr Surg Int 1997;12(2-3):81-5.

9. Springer A, et al. An update of current practice in hypospadias surgery. DOI: https://doi.org/10.1016/j.eursup.2016.09.006

10. Appeadu-Mensah W, et al. Complications of hypospadias surgery: Experience in a tertiary hospital of a developing country. http://www.afrjpaedsurg.org/text. asp?2015/12/4/211/172538

11. Snodgrass WT, Lorenzo A. Tubularized incised plate urethroplasty for proximal hypospadias. BJU Int 2000;86:1050-3.

12. Snodgrass WT. Tubularized, incised plate urethroplasty for distal hypospadias. J Urol 1994;151(2):464-5.

13. Long $\mathrm{CJ}$, et al. Intermediate-term followup of proximal hypospadias repair reveals high complication rate. DOI: https:// doi.org/10.1016/j.juro.2016.11.054

14. Dindo D, et al. Classification of surgical complications: a new proposal with evaluation in a cohort of 6336 patients and results of a survey. DOI: 10.1097/01.sla.0000133083.54934.ae

15. Hayashi Y, Kojima Y. Current concepts in hypospadias surgery. https://doi.org/10.1111/j.1442-2042.2008.02081.x

16. Bhat A, Mandal AK. Acute postoperative complications of hypospadias repair. Indian J Urol 2008;24(2):241-8.

17. Long CJ, Canning DA. Proximal hypospadias: we aren't always keeping our promises. DOI: 10.12688/f1000research.9230.1

18. Larrinaga-Simon J, et al. Tratamiento quirúrgico del hipospadias. Nuestra experiencia y seguimiento durante el período 1993-1998. Arch Esp Urol 2000;2(53):155-8.

19. Spinoit AF, et al. Hypospadias repair at a tertiary care center: Long-term followup is mandatory to determine the real complication rate. DOI: http://dx.doi.org/10.1016/j. juro.2012.12.100

20. Elbakry A. Further experience with the tubularized-incised urethral plate technique for hypospadias repair. BJU Int 2002;89(3):291-4.

21. Khan M, et al. Hypospadias Repair: A Single Centre Experience. DOI: http://dx.doi.org/10.1155/2014/453039

22. Snodgrass $W T$, Lorenzo A. Tubularized incised-plate urethroplasty for hypospadias reoperation. BJU Int. 2002;89(1):98-100.

23. Milicevic R, et al. Our Experience with tubularized incised plate urethroplasty for distal and mid-penile hypospadias. Ada Media Medianae 2003;42:35-37.

24. Steven $L$, et al. Current practice in paediatric hypospadias surgery; A specialist survey. DOI: https://doi.org/10.1016/j. jpurol.2013.04.008

25. Samuel M, et al. Distal hypospadias: Which repair? BJU Int 2002;90(1):88-91.

26. Mousavi SA, et al. Tubularized incised plate urethroplasty for hypospadias reoperation: A review and meta-analysis. Int Braz J Urol 2014;40(5):588-95.

27. Maarouf AM, et al. Single vs. double dartos layers for preventing fistula in a tubularised incised-plate repair of distal hypospadias. DOI: https://doi.org/10.1016/j. aju.2012.09.002

28. American Academy of Pediatrics. Timing of elective surgery on the genitalia of male children with particular reference to the risks, benefits, and psychological effects of surgery and anesthesia [en línea]. Dirección URL: http://pediatrics. aappublications.org/content/pediatrics/97/4/590.full.pdf.

29. Tekgul S, et al. EAU Guidelines on Paediatric Urology. DOI: https://doi.org/10.1016/j.eururo.2012.05.059

30. Perlmutter $A E$, et al. Impact of patient age on distal hypospadias repair: A surgical perspective. DOI: https://doi. org/10.1016/j.urology.2006.03.079

31. Bhat A. General considerations in hypospadias surgery. Indian J Urol 2008;24(2):188-94. 